\title{
Methodology Report \\ Simple and Specific Dual-Wavelength Excitable Dye Staining for Glycoprotein Detection in Polyacrylamide Gels and Its Application in Glycoproteomics
}

\author{
Yu-Hsuan Chiang, ${ }^{1}$ Yu-Jen Wu, ${ }^{2}$ Ya-Ting Lu, ${ }^{3}$ Kuan-Hung Chen, ${ }^{3}$ Tzu-Chun Lin, ${ }^{4}$ \\ Yu-Kuang H. Chen, ${ }^{5}$ Ding-Tzai Li, ${ }^{3}$ Fong-Ku Shi, ${ }^{3}$ Ching-Chuan Chen, ${ }^{4}$ and Jue-Liang Hsu ${ }^{1}$ \\ ${ }^{1}$ Graduate Institute of Biotechnology, National Pingtung University of Science and Technology, Pingtung 91201, Taiwan \\ ${ }^{2}$ Department of Beauty Science, Meiho University of Technology, Pingtung 91202, Taiwan \\ ${ }^{3}$ Mass Solutions Technology Co. Ltd., New Taipei City 22101, Taiwan \\ ${ }^{4}$ Food Science and Nutrition Department, Meiho University of Science and Technology, Pingtung 91202, Taiwan \\ ${ }^{5}$ Department of Plant Industry, National Pingtung University of Science and Technology, Pingtung 91201, Taiwan \\ Correspondence should be addressed to Jue-Liang Hsu, jlhsu@mail.npust.edu.tw
}

Received 20 May 2011; Accepted 4 August 2011

Academic Editor: Susan A. Rotenberg

Copyright (C) 2011 Yu-Hsuan Chiang et al. This is an open access article distributed under the Creative Commons Attribution License, which permits unrestricted use, distribution, and reproduction in any medium, provided the original work is properly cited.

In this study, a commercially available fluorescent dye, Lissamine rhodamine B sulfonyl hydrazine (LRSH), was designed to specifically stain the glycoproteins in polyacrylamide gels. Through the periodate/Schiff base mechanism, the fluorescent dye readily attaches to glycoproteins and the fluorescence can be simultaneously observed under either $305 \mathrm{~nm}$ or $532 \mathrm{~nm}$ excitation therefore, the dye-stained glycoproteins can be detected under a regular UV transilluminator or a more elegant laser-based gel scanner. The specificity and detection limit were examined using a standard protein mixture in polyacrylamide gels in this study. The application of this glycoprotein stain dye was further demonstrated using pregnancy urine samples. The fluorescent spots were further digested in gel and their identities confirmed through LC-MS/MS analysis and database searching. In addition, the Nglycosylation sites of LRSH-labeled uromodulin were readily mapped via in-gel PNGaseF deglycosylation and LC-MS/MS analysis, which indicated that this fluorescent dye labeling does not interfere with enzymatic deglycosylation. Hence, the application of this simple and specific dual-wavelength excitable dye staining in current glycoproteome research is promising.

\section{Introduction}

Most eukaryotic proteins are posttranslationally glycosylated at Asn residues with $\mathrm{N}$-linked glycans, at Ser/Thr residues with O-linked glycans, or at protein C-terminal carboxylic acid with glycosyl phosphatidylinositol (GPI) anchors [1-3]. These glycosylations can alter a protein's physical properties such as steric hindrance, charged state, or hydrophobicity, which contribute to the activity, recognition, immunogenicity, solubility, and stability of proteins [4]. Therefore, many physiological processes such as cell growth control, cell migration, cell adhesiveness, tissue differentiation, and inflammatory reactions are closely associated with protein glycosylation $[5,6]$. Among the current strategies for proteome study, 2D gel electrophoresis is one of the most power- ful tools for separating proteins from complex mixtures. Using this approach, large-scale protein expression profiling can be performed by direct comparison with protein spots stained by Coomassie blue, silver, or some fluorescent dyes on two individual gels. Then, protein spots of interest can be digested in gel and identified by mass spectrometry (MS) and database matching. For comprehensive proteomic study, the analysis of protein posttranslational modifications (PTMs) has become an important issue. Direct observation of the protein PTMs in gels will not only conveniently provide the location of modified proteins in the gel but will also afford useful information about their relative abundances. Therefore, specific staining dyes for PTM monitoring have attracted much attention, and some have been developed and are widely applied in current proteome studies in order to 
detect proteins with PTMs in gels. For example, Pro-Q Diamond developed by Molecular Probes (Invitrogen detection technologies) is now a popular fluorescent staining kit for phosphoprotein detection. Protein phosphorylations on Ser, Thr or Tyr residues can be directly visualized in gel with a high sensitivity and are compatible with MS-based protein identification [7]. For glycoprotein detection, there are two main strategies widely used for the detection of glycoproteins in gels or on blots. The first method is based on affinity interaction between carbohydrate epitopes and lectin incorporated with reporter tags such as fluorescent or chemiluminescent substrates for downstream detection [8-10], and the second approach is to covalently react carbohydrate groups with hydrazine moieties through a periodate/Schiff base (PAS) mechanism. The PAS-based approach can be used to label all kinds of glycoproteins bearing cis-vicinal diols on carbohydrates without the specific recognition toward carbohydrate epitopes therefore this approach can feasibly be used for global glycoprotein detection. The labeling chemistry of the PAS-based approach involves dialdehyde formation via oxidative cleavage of cis-vicinal diols on carbohydrates followed by the coupling of hydrazide reporters with aldehydes via Schiff base formation. The Schiff bases or their reduced forms can emit luminescence or fluorescence that can be readily detected by some optical devices such as CCD cameras or laser image scanners. Luminescent staining can be achieved via PAS attachment of digoxigenin hydrazide (or biotin hydrazide) on carbohydrates, followed by immunoaffinity adsorption of antidigoxigenin (or streptavidin) conjugated with alkaline phosphatase or horseradish peroxidase, and in the presence of chemiluminescent substrates, highly sensitive luminescence occurs and acts as a location indicator of glycoproteins $[11,12]$. However, this luminescence staining is usually applied to electroblot membranes and its high cost and tedious procedure may limit its application [13]. Alternatively, fluorescent staining, in which chromogenic substrates (such as acid fuchsin or Alcian Blue) $[14,15]$ as well as fluorescent dyes are directly incorporated into the carbohydrate during the PAS reaction $[13,16$, 17], may circumvent some of the problems derived from immunoblotting, such as membrane transfer procedure and high cost, providing a convenient method for glycoprotein detection in gels. Among these fluorescent staining methods, Pro-Q Emerald 300 and Pro-Q Emerald 480, which can be excited in the UV and visible light region, respectively, have been shown to be well suited for gel-based glycoprotein detection in terms of sensitivity and convenience [13, 17-19].

In this study, we utilized Lissamine Rhodamine B sulfonyl hydrazine (LRSH) derived from readily available Rhodamine B sulfonyl chloride to label the glycoproteins in gel via the PAS mechanism. Using this dye, glycoproteins can be readily labeled and detected in gel under a suitable gel imager. According to the UV-visible absorption spectrum of this dye, a $\lambda_{\max }$ at $557 \mathrm{~nm}$ accompanied by a minor absorption at UV $305 \mathrm{~nm}$ were simultaneously observed, which implied that glycoprotein detection can be performed on either regular UV transilluminators or more elegant laserbased gel scanners. Some standard glycoproteins separated by SDS-PAGE were employed in this study to evaluate the efficiency of this dual wavelength excitable dye in terms of sensitivity and specificity. Urine glycoproteins resolved by SDS-PAGE and 2-DE were detected by this approach, and the spots of interest were further digested in gel and the identities and glycosylation sites of the glycoproteins characterized by LC-MS/MS experiments for the assessment of this method's compatibility with the current protein identification workflow.

\section{Materials and Methods}

2.1. Materials. Tris-base, Coomassie blue, acetonitrile (ACN), 2-Mercaptoethanol, sodium acetate, acetic acid, methanol, N,N-dimethylformamide, ammonium bicarbonate, and 1,4-dithiothreitol (DTT) were purchased from J. T. Baker (Phillipsburg, NJ, USA). Glycine, SDS (sodium dodecyl sulfate), formic acid (FA), AGP ( $\alpha 1$-acid glycoprotein from human plasma), fetuin (from fetal calf serum), BSA (bovine serum albumin), HRP (horseradish peroxidase), IgG (human immunoglobulin G), RNase B (ribonuclease B from bovine pancreas), hydrazine (1 M in tetrahydrofuran), trifluoroacetic acid (TFA), and iodoacetamide (IAM) were obtained from Sigma-Aldrich (MO, USA). Bromophenol Blue and sodium cyanoborohydride were purchased from Riedel-de Haën (Seelze, Germany). Lissamine rhodamine B sulfonyl chloride was purchased from Acros Organics (PA, USA). Sodium metaperiodate was purchased from Alfa Aesar (Lancashire, UK). Sequencing grade modified trypsin was obtained from Promega (WI, USA). PNGase F was purchased from New England BioLabs (MA, USA). Prestained protein marker was purchased from Yeastern Biotech (Taipei, Taiwan).

2.2. Preparation of LRSH. Lissamine rhodamine B sulfonyl chloride $(29 \mathrm{mg}, 0.05 \mathrm{mmol})$ was dissolved in anhydrous DMF $(500 \mu \mathrm{L})$ then $100 \mu \mathrm{L}$ of anhydrous hydrazine $(1 \mathrm{M}$ in tetrahydrofuran) was added. The reaction mixture was vortexed at $\mathrm{rt}$ in the dark for $3 \mathrm{~h}$, then the solvent and excess hydrazine were removed by SpeedVac. The resulting Lissamine rhodamine B sulfonyl hydrazine (LRSH) was stored at $-20^{\circ} \mathrm{C}$ prior to the glycoprotein staining experiment. The absorption and emission wavelengths of LRSH were obtained using a Hitachi F-4500 fluorescence spectrophotometer (Hitachi Instruments, Tokyo, Japan).

2.3. Sample Preparation. The sample preparation of urine samples from pregnant women was based on the uromodulin enrichment procedure reported previously [19], but with some modifications. Briefly, $1 \mathrm{~mL}$ of pregnancy urine was centrifuged at $10000 \times \mathrm{g}$ at $4^{\circ} \mathrm{C}$ for $1 \mathrm{~h}$. The supernatant was removed and the precipitate was dissolved in sample buffer for SDS-PAGE. For the 2-DE experiment, $10 \mathrm{~mL}$ of pregnancy urine was fractionated in a similar manner, and the precipitate was dissolved in sample buffer for IEF separation.

2.4. Gel Electrophoresis. For SDS-PAGE, the protein sample was mixed with equal volume of sample buffer containing $100 \mathrm{mM}$ Tris ( $\mathrm{pH}$ 6.8), 4\% SDS, 20\% glycerol, $40 \mathrm{mM}$ 
2-mercaptoethanol, and $0.2 \%$ bromophenol blue. After denaturation, the mixture was loaded onto SDS polyacrylamide linear gradient gel which was polymerized from a combination of $10 \% \mathrm{~T}$ and $2.6 \% \mathrm{C}(\% \mathrm{~T}$ is the total monomer concentration expressed in grams per $100 \mathrm{~mL}$, and \%C is the percentage cross-linker). After stacking on the upper gel layer, proteins were separated by electrophoresis which was carried out at $30 \mathrm{~V}$ in a running buffer $(25 \mathrm{mM}$ Tri-base, $20 \mathrm{mM}$ Glycine, $0.1 \% \mathrm{w} / \mathrm{v}$ SDS) until the dye front reached the end of the gel. In this study, broad range molecular weight prestained protein markers $(240-7 \mathrm{kDa})$ (Yeastern Biotech, Taiwan) were used for the protein size detection. Among these marker proteins, protein spots at 70 and $25 \mathrm{kDa}$ were fluorescent and were also observable upon UV or visible light excitation (orange color). For 2D gel experiments, the first dimension electrophoresis (isoelectric focusing) was performed on a Bio-Rad PROTEAN IEF Cell at $20^{\circ} \mathrm{C}$ with a current limit of $50 \mathrm{~A}$ per strip. A sample was dissolved in a rehydration buffer and applied on an IPG strip in a strip holder. Every 11-cm IPG strip (pI 4-7, Immobiline DryStrip) was rehydrated at $50 \mathrm{~V}$ for $12 \mathrm{~h}$, and the protein mixture was focused according to the preset program: $200 \mathrm{~V}(1.5 \mathrm{~h})$, $500 \mathrm{~V}(1 \mathrm{~h}), 1,000 \mathrm{~V}(1 \mathrm{~h}), 4,000 \mathrm{~V}(1 \mathrm{~h}), 8,000 \mathrm{~V}(2 \mathrm{~h})$, until the total Vh reached 19,960. After isoelectric focusing, the strip was removed and equilibrated in $5 \mathrm{~mL}$ equilibration buffer (50 mM Tris-HCl, pH 8.8, $6 \mathrm{M}$ urea, 30\% glycerol, $2 \%$ SDS, $0.01 \%$ bromophenol blue) containing $1 \%(\mathrm{w} / \mathrm{v})$ DTT for $10 \mathrm{~min}$, then transferred to another $5 \mathrm{~mL}$ equilibration buffer containing $2.5 \%(\mathrm{w} / \mathrm{v})$ iodoacetamide for $10 \mathrm{~min}$. The equilibrated strip was placed on the top of a SDS-PAGE gel $(12.5 \%)$ and sealed with $0.5 \%$ agarose, then the second dimension electrophoresis was run at $150 \mathrm{~V}$ for $6.5 \mathrm{~h}$.

\subsection{Staining and Detection of LRSH-Conjugated Glycopro-} teins. The gel obtained from either the SDS-PAGE or 2DGE experiments was fixed in $50 \mathrm{~mL}$ of fixed buffer $(40 \%$ methanol and $7 \%$ acetic acid) for $1 \mathrm{~h}$, and the gel was stained by LRSH via a similar procedure as PAS. Briefly, the gel was immersed in $50 \mathrm{~mL}$ of oxidation buffer $(10 \mathrm{mg}$ of sodium periodate in 3\% acetic acid) and gently shaken for $1 \mathrm{~h}$ in the dark. The excess sodium periodate was then washed away by $3 \%$ acetic acid $(50 \mathrm{~mL} \times 2)$. The gel containing oxidized glycoproteins was completely immersed in $50 \mathrm{~mL}$ of $0.1 \mathrm{M}$ sodium acetate containing with LRSH (ca. $80 \mu \mathrm{g}$ ) and incubated by gentle agitation for $40 \mathrm{~min}$ in the dark. The gel slide was washed in $50 \mathrm{~mL}$ of destaining solution (containing $35 \% \mathrm{ACN}, 50 \mathrm{mM}$ sodium acetate, and $1 \%$ acetic acid) with gentle agitation for $30 \mathrm{~min}$ to remove the unbound LRSH. The step was repeated two times with another $50 \mathrm{~mL}$ of destaining solution to avoid the nonspecific adsorption of this fluorescent dye. Finally, the gel slide was maintained in deionized water in the dark prior to further imaging analysis. The gel image was acquired in a UV transilluminator equipped with a UV 305-nm excitation source and a CCD camera (Major Science, New Taipei City, Taiwan) or obtained using a Typhoon 9210 laser imager (GE Healthcare Life Sciences, NJ, USA). The same gel slide was poststained with Coomassie blue for differentiation between gel spots of glycoproteins and nonglycoproteins.

2.6. In-Gel Digestion of Gel Spots. The bands or spots cut from 1D or 2D gels were cut into pieces of about $1 \mathrm{~mm}$ in diameter. The gel pieces were soaked in $100 \mu \mathrm{L}$ of $50 \mathrm{mM}$ DTT in $25 \mathrm{mM}$ ammonium bicarbonate $(\mathrm{ABC})$ at $37^{\circ} \mathrm{C}$ for $1 \mathrm{~h}$. After removal of the supernatant, these gel pieces were immersed in $100 \mu \mathrm{L}$ of $100 \mathrm{mM}$ IAM in $25 \mathrm{mM} \mathrm{ABC}$ and incubated at $\mathrm{rt}$ in the dark for $30 \mathrm{~min}$. The supernatant was removed, and the Coomassie blue stain in the gel was washed with $100 \mu \mathrm{L}$ of $50 \% \mathrm{ACN}$ in $25 \mathrm{mM} \mathrm{ABC}$ under sonication several times until the Coomassie blue stain was completely removed. The gel pieces were dehydrated by soaking in ACN followed by SpeedVac concentration. The white powder gel pieces were rehydrated and immersed in $25 \mathrm{mM} \mathrm{ABC}$, and trypsin was added at a trypsin/protein weight ratio of $1 / 20$. In-gel digestion was performed at $37^{\circ} \mathrm{C}$ for $16 \mathrm{~h}$. The resulting digest was extracted cumulatively by $50 \mu \mathrm{L}$ of $50 \% \mathrm{ACN}$ in $5 \%$ TFA ten times. The combined extracts were concentrated, and the remaining tryptic digest was maintained at $-20^{\circ} \mathrm{C}$ prior to LC-MS/MS analysis. For the deglycosylation experiment, the tryptic peptides obtained from in-gel digestion were further deglycosylated by PNGase $\mathrm{F}$ in $25 \mathrm{mM} \mathrm{ABC}(\mathrm{pH} 7.5)$ at $37^{\circ} \mathrm{C}$ overnight. The reaction mixture was concentrated and maintained at $-20^{\circ} \mathrm{C}$ prior to LC-MS/MS analysis.

2.7. LC-MS/MS Analysis and Glycoprotein Identification. The resulting peptide mixture derived from in-gel digestion of LRSH-labeled glycoprotein was separated using a Surveyor HPLC system (ThermoFinnigan) utilizing a $75 \mu \mathrm{m}$ i.d. capillary column packed with $5-\mu \mathrm{m}$ particles (MST, Taiwan) with a linear gradient from $5 \%$ to $60 \%$ acetonitrile containing $0.1 \%$ formic acid over $60 \mathrm{~min}$. The separated peptides were analyzed online on a LCQ DECA XP MAX ion trap mass spectrometer (ThermoFinnigan, CA, USA) equipped with a nano ESI source (ThermoFinnigan). The MS/MS spectra were acquired in a data-dependent mode that determined the masses of the positive parent ions and fragments of the three strongest ions. The scan range was from m/z 400 to 1600 for MS and m/z 50 to 2000 for MS/MS. All MS/MS spectra were processed using MASCOT Distiller (Matrix Science, UK), and the resulting MGF file was searched using the Mascot search engine v2.2 (Matrix Science, UK) with the following search parameters: (1) protein database was set to be SwissProt; (2) taxonomy was set as Homo sapiens (human); (3) one trypsin missed cleavage was allowed; (4) the mass tolerance was set at $1.5 \mathrm{Da}$ for the precursor and $0.8 \mathrm{Da}$ for the product ions; (5) carbamidomethyl (C) was chosen for fixed modification; (6) oxidation (M) and deamidated (NQ) were chosen for variable modifications (herein, the variable modification of deamidated (NQ) was used to determine the N-glycosylation sites); (7) proteins with scores above the significance threshold $(P<0.05)$ were shown as significant hits. The hit with the highest score which contained at least two peptides with scores beyond the identity threshold was regarded as the identified protein from each gel spot. All 


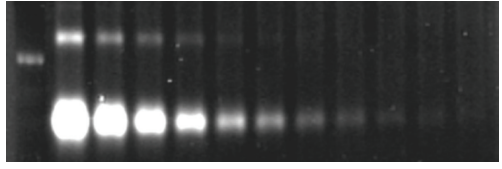

$\begin{array}{llllllllllll}M & 1 & 2 & 3 & 4 & 5 & 6 & 7 & 8 & 9 & 10 & 11\end{array}$

(a)

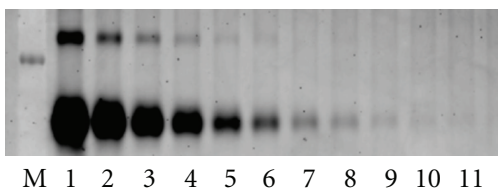

(b)

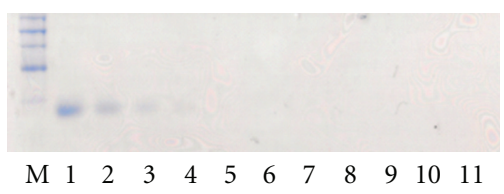

(c)

FIGURE 1: Detection of LRSH-labeled $\alpha 1$-acid glycoproteins in SDS-polyacrylamide gel with different imagers and poststaining with Coomassie blue. Twofold serial dilutions of the glycoproteins, ranging from $1 \mu \mathrm{g}$ to $0.95 \mathrm{ng}$, are displayed in Lane 1 to Lane 11 . $\mathrm{M}$ denotes molecular weight markers. (a) UV 305-nm excitation and CCD camera detection in a UV transilluminator. (b) 532$\mathrm{nm}$ excitation and detection in a Typhoon 9210 laser scanner. (c) Poststaining of the same gel with Coomassie blue.

MS/MS spectra of identified peptides were further verified by manual interpretation.

\section{Results and Discussions}

3.1. In-Gel Glycoprotein Detection by Postlabeling with LRSH. Lissamine Rhodamine B sulfonyl hydrazine ( $\mathrm{LRSH}$ ) is readily available from commercial sources or can be prepared simply from the reaction of rhodamine $B$ sulfonyl chloride with hydrazine. LRSH is a hydrazide derivative of sulforhodamine $B$ that can spontaneously react with aldehyde moieties generated from glycans on glycoproteins via oxidative cleavage by sodium periodate. It has been widely used as a probe to label cell surface glycoproteins or glycoproteins in solution but not in polyacrylamide gel [20]. Its feasibility for glycoprotein detection in gel was examined in this study. The UV-visible spectrum of LRSH showed that an excitation maximum at a wavelength of $557 \mathrm{~nm}$ was observed and accompanied by a weak absorption band at $303 \mathrm{~nm}$ when dissolved in methanol (data was shown in Figure S1 of Supplementary Material available at doi 10.1155/2011/780108). A fluorescence emission at around $588 \mathrm{~nm}$ can be simultaneously obtained when LRSH is irradiated at either $557 \mathrm{~nm}$ or $303 \mathrm{~nm}$. Regular UV transilluminators equipped with 305nm UV light excitation system, widely used for DNA gel imaging, were able to be utilized for the detection of LRSHlabeled glycoprotein in gel. Due to the lack of an excitation source of $557 \mathrm{~nm}$ in our laser-based gel scanner Typhoon 9210 , a close green laser $(532 \mathrm{~nm})$ source was used in this

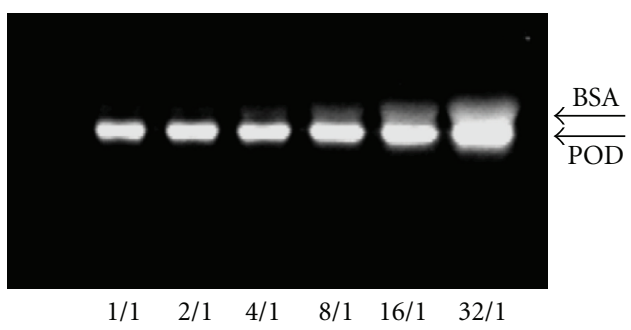

(a)

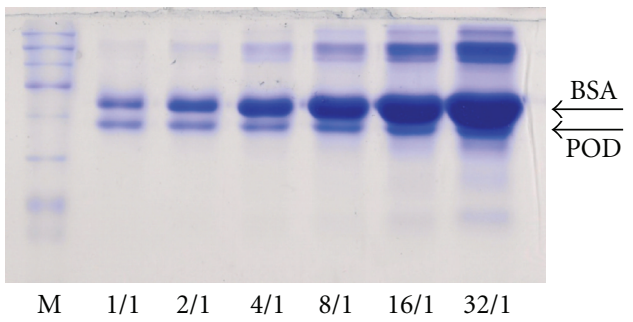

(b)

FIGURE 2: Specificity testing of LRSH glycoprotein stain dye. Twofold serial increases in the nonglycoprotein (BSA)/glycoprotein (horseradish peroxidase, HRP) ratio, ranging from $1 \mu \mathrm{g} / 1 \mu \mathrm{g}$ to $32 \mu \mathrm{g} / 1 \mu \mathrm{g}$. M represents standard protein molecular weight markers. (a) UV 305-nm excitation and CCD camera detection in a UV transilluminator. (b) Poststaining of the same gel with Coomassie blue.

study for the examination of LRSH's performance in laserbased gel imaging. According to the fluorescence spectra of LRSH excited at different wavelengths, we found that the fluorescence intensity excited at $532 \mathrm{~nm}$ is much more intense than that acquired at $305 \mathrm{~nm}$, as shown in Figure S2 of the Supplementary Material, which implied that superior detection limits will be obtained more from laser-based gel scanners than from UV transilluminators even though the excitation source was not the exact $\lambda_{\max }$. If the exact $\lambda_{\max }$, $557 \mathrm{~nm}$, was used as the excitation source, the fluorescent signal was more than twentyfold increase than the signal obtained under $305 \mathrm{~nm}$ excitation, which also implied that the detection limit will be further improved under the excitation of $557 \mathrm{~nm}$ wavelength. Twofold serial dilutions of AGP ( $\alpha 1$-acid glycoprotein, from human plasma) ranging from $1 \mu \mathrm{g}$ to $0.97 \mathrm{ng}$ in SDS polyacrylamide gel were stained with LRSH via the PAS mechanism. Images of the same gel acquired using the UV transilluminator $(305 \mathrm{~nm})$, the Typhoon 9210, and poststained with Coomassie blue are shown in Figure 1, respectively. The detection limit of the glycoprotein ( $\alpha 1$-acid glycoprotein) was around $1 \mathrm{ng}$ using either the UV transilluminator $(305 \mathrm{~nm})$ or the Typhoon 9210, and both detection methods were much more sensitive than Coomassie blue staining. In comparison with two commercial staining kits, the results showed that the sensitivity of LRSH toward $\alpha 1$-acid glycoprotein was slightly poorer than Pro-Q Emerald 300 dye (300 pg) (the data was shown in Figure S3 of Supplementary Material) but slightly superior to Pro-Q Emerald 488 dye (4.7 ng) (according to the report in [17]) toward the same glycoprotein [13,17]. Due to 


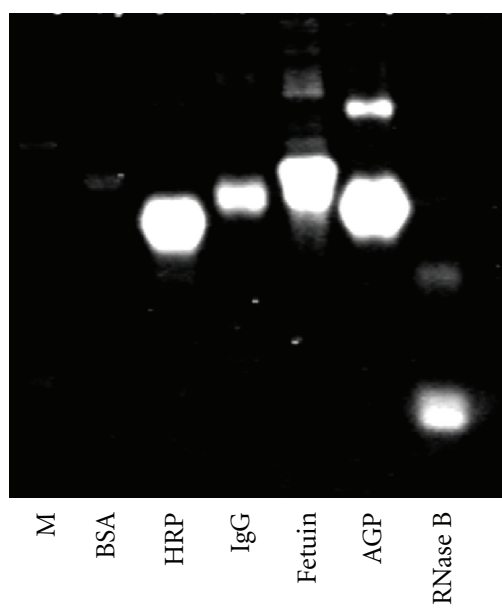

(a)

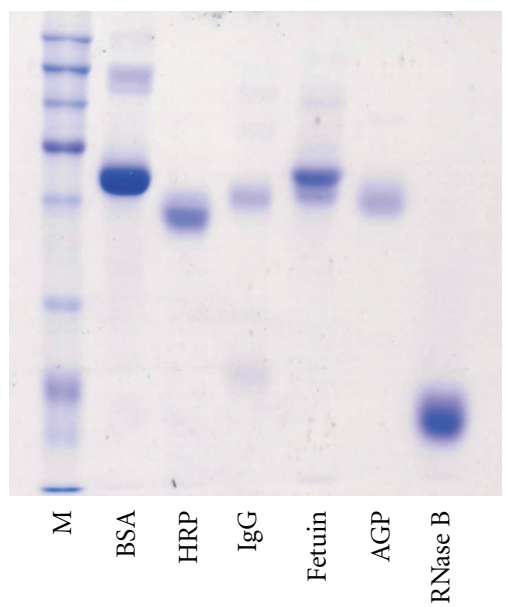

(b)

Figure 3: (a) LRSH staining of various glycoproteins. (b) Poststained with Coomassie blue. Each lane was loaded with two $\mu \mathrm{g}$ of protein. $\mathrm{M}$ represents standard protein molecular weight markers. Bovine serum albumin (nonglycoprotein), horseradish peroxidase, immunoglobin G, $\alpha$-acid glycoprotein, and ribonuclease B are denoted by BSA, HRP, IgG, AGP, and RNase B.

the reason that UV $305 \mathrm{~nm}$ is not the absorption $\lambda_{\max }$ of LRSH, the detection limit of LRSH is expected to be dramatically improved when this LRSH-stained gel is excited at $557 \mathrm{~nm}$, and the resulting emission is detected at $588 \mathrm{~nm}$. Meanwhile, the linearity correlation between glycoprotein amounts and their fluorescence responses was also examined in this study by UVItec (Cambridge, UK) gel image quantitation software. An $R^{2}$ value of 0.97 was obtained between these concentration ranges, which suggested that the quantitative analysis of the same glycoprotein in gel by this approach was trustable (the data was shown in Figure S4 of Supplementary Material).

The specificity of LRSH toward glycoproteins was also examined using the samples with higher nonglycoprotein (BSA)/glycoprotein (horseradish peroxidase) ratios. Under UV 305-nm excitation, the staining specificity was as shown in Figure 2, the samples with BSA/peroxidase ratios ranging

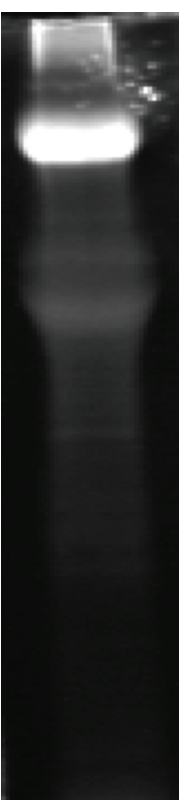

(a)

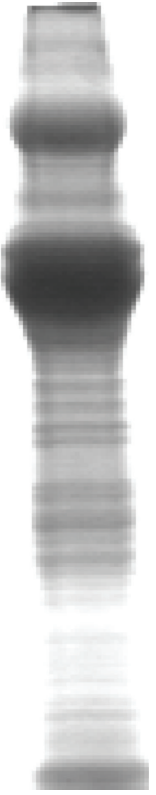

(b)
FIGURE 4: (a) LRSH staining of prefractionated pregnancy urine in SDS-PAGE. (b) Poststained with Coomassie blue. A total of $1 \mathrm{~mL}$ of pregnancy urine was utilized for prefractionation and SDS-PAGE analysis.

from $1 / 1$ to $32 / 1$, and the amount of peroxidase was fixed to $1 \mu \mathrm{g}$. Figure $2(\mathrm{a})$ shows the fluorescent image acquired in a UV transilluminator $(305 \mathrm{~nm})$ and Figure 2(b) shows the same gel poststained with Coomassie blue. This result indicated that nonspecific staining was not apparent, and weak BSA band was only observed when the nonglycoprotein/glycoprotein ratio was greater than $8 / 1$. This may be caused by the intrinsic protein fluorescence rather than nonspecific adsorption according to the paper presented by Zhao et al. [21]. According to Zhao's report, the intrinsic fluorescence of BSA becomes apparent in amounts greater than a couple of micrograms in a solution containing 1$10 \%$ acetic acid and $40 \%$ methanol. Although the intrinsic fluorescence of BSA became observable at amounts greater than $16 \mu \mathrm{g}$, its fluorescence was still much less intensive than the signal poststained by Coomassie blue, as shown in Figure 2. This result implied that the intrinsic fluorescence caused by highly abundant nonglycoproteins in real cases will not interfere with the in-gel glycoprotein determination by LRSH staining when the LRSH-stained and Coomassie blue poststained gels are compared. Similar result was obtained when Pro-Q Emerald 300 dye was performed at the same experiment (data was shown in Figure S5 of Supplementary Material), which implied that the specificity of LRSH is comparable with Pro-Q Emerald 300 dye.

The fluorescence intensity can directly reflect the amount of the same glycoprotein, as indicated in the above-mentioned linearity assay (data shown in Figure 4 of Supplementary Material); however, the glycan content of a protein is also a factor correlated with the fluorescence intensity 


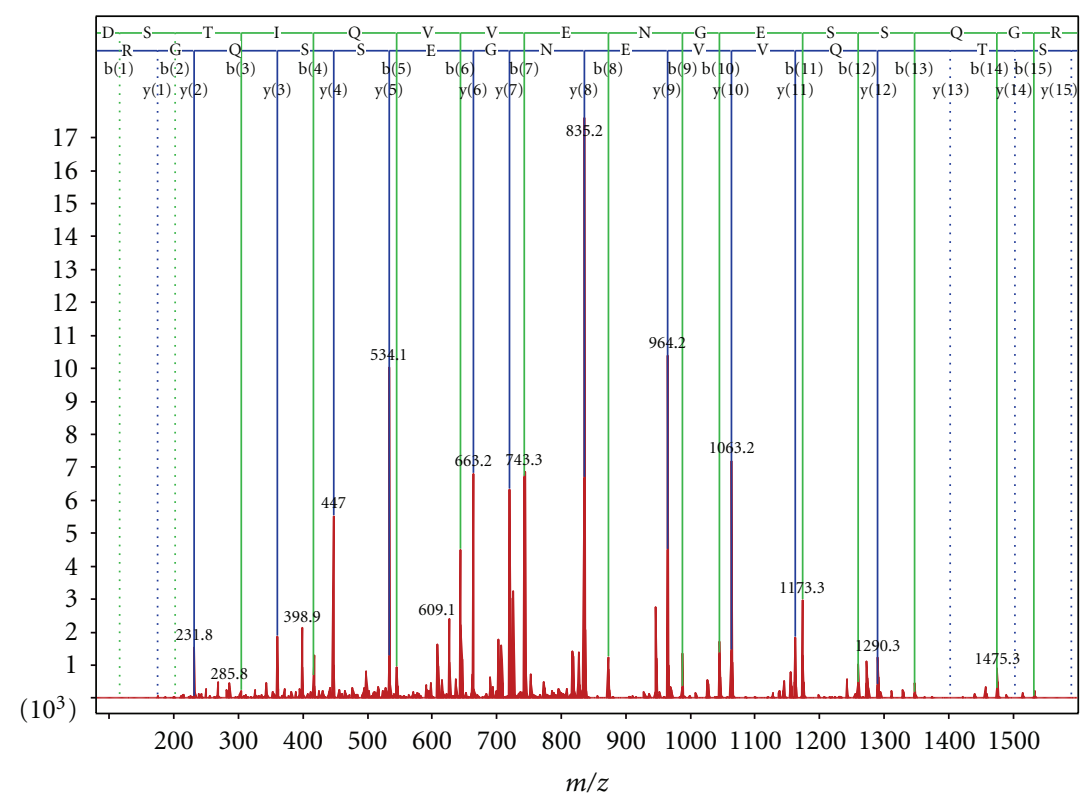

FIGURE 5: A representative MS/MS spectrum of peptide DSTIQVVENGESSQGR derived from uromodulin. The most intensive spot on SDS-PAGE in Figure 4 was in-gel digested and analyzed by LC-MS/MS.

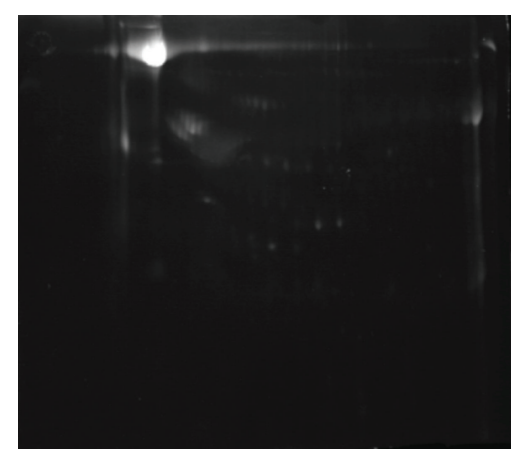

(a)

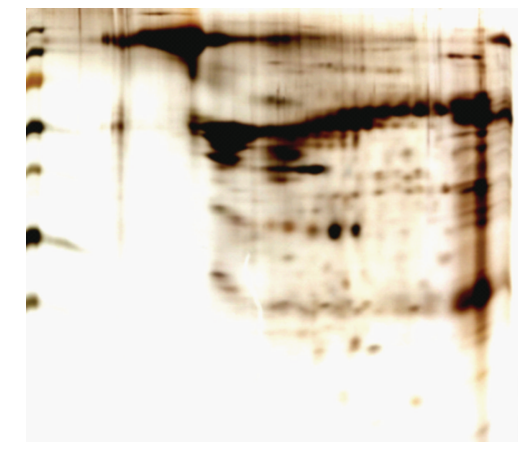

(b)

FIGURE 6: (a) LRSH staining of prefractionated pregnancy urine in 2-DE. (b) Silver staining of the same gel. A total of $10 \mathrm{~mL}$ of pregnancy urine was utilized for prefractionation and 2-DE analysis.

of LRSH-labeled glycoprotein. Some glycoproteins with different glycan contents were simultaneously analyzed in the same gel to evaluate the fluorescence response towards glycan content. $1 \mu \mathrm{g}$ of BSA, horseradish peroxidase (HRP), human immunoglobulin $\mathrm{G}$ (IgG), fetuin, $\alpha 1$-acid glycoprotein from human plasma (AGP), and ribonuclease B from bovine pancreas (RNase B) were loaded in parallel lanes and separated by SDS-PAGE. The fluorescence image of the LRSHlabeled gel acquired using the UV transilluminator $(305 \mathrm{~nm})$ is shown in Figure 3(a), and Figure 3(b) shows the image of the same gel under Poststaining with Coomassie blue. Apparently, the fluorescence intensities of all glycoproteins with various carbohydrate contents were much more intensive than the intensities stained with Coomassie blue, and the trend of fluorescence intensity was closely correlated to the carbohydrate content of each glycoprotein, which was similar to the result demonstrated by Steinberg et al. [13].
3.2. The Detection of Glycoproteins from Urine Samples in SDS-PAGE and 2-DE Gels. In order to demonstrate the applicability of LRSH in complex biological samples, pregnancy urine was utilized for in-gel glycoprotein staining. An uromodulin-rich fraction was prepared from $1 \mathrm{~mL}$ of pregnancy urine according to the protocol presented by Easton et al. [22]. The protein mixture in this fraction was separated by SDS-PAGE then stained with LRSH for the purpose of characterizing the location of uromodulin in gel. As shown in Figure 4(a), only one intensive fluorescent band was observed in this gel, which suggested that the most abundant glycoprotein in this crude fraction could be uromodulin, an 85-kDa glycoprotein frequently observed in human pregnancy urine. The total protein distribution was displayed in the same gel poststained with Coomassie blue, as shown in Figure 4(b). By comparison of these two gel images, the locations of glycoproteins in this SDS-PAGE and the 


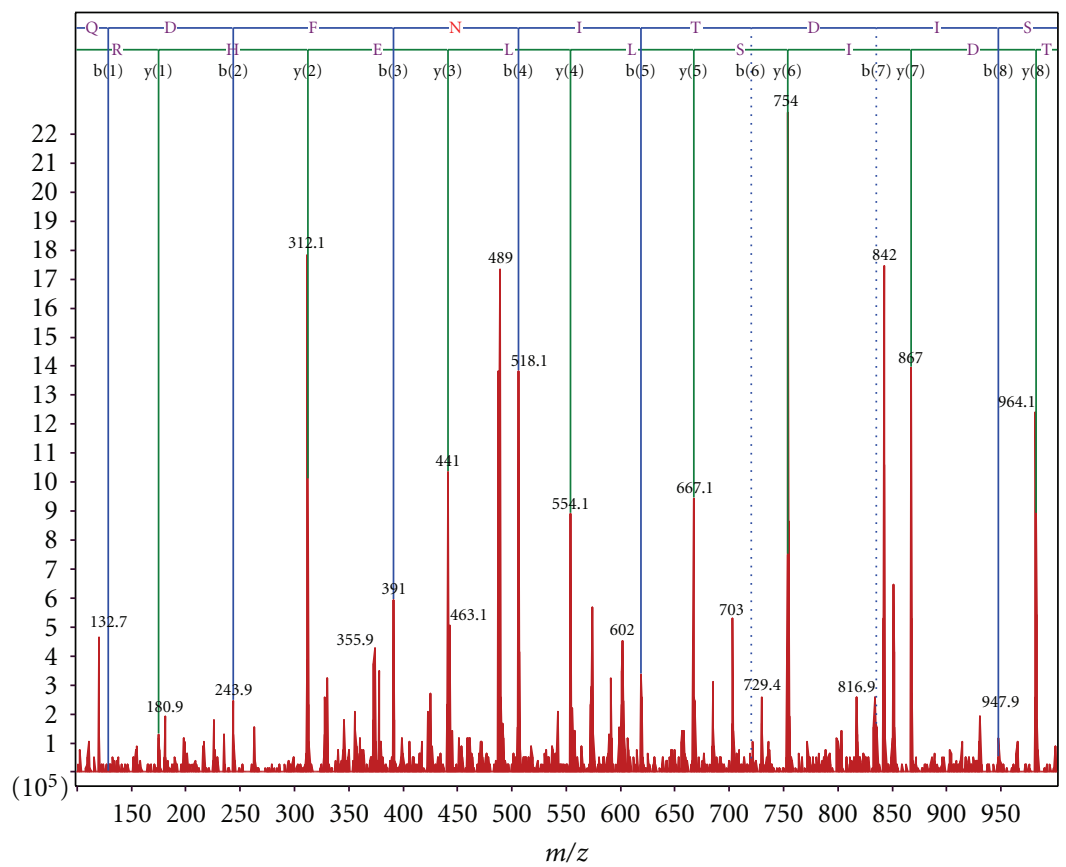

FIgURE 7: MS/MS spectrum of the deglycosylated peptide QDFNITDISLLEHR. N represents the Asp residue formed via PNGase F deglycosylation from the glycosylation site at the Asn residue.

purification performance can be determined. The identity of this major glycoprotein was further confirmed by in-gel digestion and LC-MS/MS analysis. Through Mascot database searching, fifteen tryptic peptides belonging to uromodulin were characterized (shown in Table S1 of Supplementary Material) and the MS/MS spectrum of a represented peptide DSTIQVVENGESSQGR is shown in Figure 5. This result also indicated that this dye labeling will not interfere with the in-gel trypsin digestion toward LRSH-labeled glycoprotein and the downstream protein identification. The same pregnancy urine was also separated by 2-DE then stained by LRSH followed by Poststaining with silver. The glycoproteinspecific gel image and the silver-stained gel image are shown in Figures 6(a) and 6(b), respectively. After in-gel digestion, LC-MS/MS analysis and Mascot database searching, similar results as for the SDS-PAGE experiment were obtained, and the major spot in Figure 6(a) was identified as uromodulin. In addition, the tryptic digest of the fluorescent gel band was further deglycosylated by PNGase F, and the resulting peptides bearing $\mathrm{N}$-glycosylation sites were further analyzed by LC-MS/MS. The glycosylation site was recognized by a variable modification setting deamidated (NQ) (Asn $\rightarrow$ Asp) with a 1-Da molecular weight difference at the Asn residue through Mascot database searching. Two deglycosylated peptides derived from uromodulin were identified and their $\mathrm{N}$-glycosylation sites were assigned as ${ }^{322} \mathrm{~N}$ and ${ }^{396} \mathrm{~N}$, respectively. These assignments were consistent with the N-glycosylation sites of uromodulin recorded in the UniProt database (http://www.uniprot.org/). The MS/MS assignment of the deglycosylated peptide QDFNITDISLLEHR, which contained the glycosylation site ${ }^{322} \mathrm{~N}$, is illustrated in Figure 7. The MS/MS spectrum of the other deglycosylated peptide was as shown in Figure S5 of Supplementary Material. These results indicated that the chemical modification of glycan and fluorescent dye labeling does not interfere with PNGase $\mathrm{F}$ deglycosylation and the downstream $\mathrm{N}$-glycosylation mapping. Hence, this in-gel glycoprotein staining is not only compatible with the gel-based analytical pipeline in current proteome research but feasible for the mapping of glycosylation sites.

\section{Concluding Remarks}

LRSH fluorescent dye is available commercially or can be simply prepared via the reaction of rhodamine B sulfonyl chloride with hydrazine. This simple and dual-wavelength excitable dye can label the glycoproteins in gel easily, and the fluorescent emission of LRSH-labeled glycoproteins can be readily detected on either regular UV transilluminators or elegant laser-based gel scanners, which provides convenience and flexibility for various applications. Its nano-gram level detection limit and high specificity towards glycoproteins in either SDS-PAGE or 2-DE are feasible to current glycoproteome research. By comparing with two commercial staining kits, the sensitivity of LRSH toward $\alpha 1$-acid glycoprotein (ca. $1 \mathrm{ng}$ ) is slightly poorer than Pro-Q Emerald 300 dye (300 pg) (the data was shown in Figure S3 of Supplementary Material) but slightly superior to Pro-Q Emerald 488 dye $(4.7 \mathrm{ng})$ (according to the report in [17]) toward the same glycoprotein. The specificity of LRSH stain dye is comparable to Pro-Q Emerald 300 according to the data shown in Figure S5 of Supplementary Material. The operation steps and time required for LRSH staining are 5 steps in about $4 \mathrm{~h}$, which are also comparable to either Pro-Q Emerald 300 or Pro-Q 
Emerald 488 (7 steps in $4 \mathrm{~h}$ ) $[13,17]$. In addition, the dualwavelength excitable stain dye can be detected on regular UV transilluminators (UV $305 \mathrm{~nm}$ ) and laser-based gel scanners $(488 \mathrm{~nm})$ with similar detection limits, which is more flexible than Pro-Q Emerald 300 or Pro-Q Emerald 488 in terms of the availability of detection instrument. Therefore, this simple and home-made LRSH dye can be utilized for glycoprotein staining without using commercial ProQ Emerald 300 and Pro-Q Emerald 488 kits. Using LRSH glycoprotein stain dye, glycoproteins can be easily observed, and the identities of these glycoproteins can be readily recognized through in-gel digestion, LC-MS/MS analysis, and database matching. Uromodulin from pregnancy urine can be readily observed and identified. Remarkably, two Nglycosylation sites of uromodulin were characterized through this approach, which indicated that the LRSH labeling on carbohydrates in glycoproteins does not interfere with the enzymatic deglycosylation therefore, this in-gel glycoprotein staining dye is feasible for the current glycoproteome analysis.

\section{Authors' Contribution}

Yu-Hsuan Chiang, Yu-Jen Wu, and Ya-Ting Lu contributed equally to this work and they share the first author position equally.

\section{Acknowledgment}

This work was supported by a Taiwan NSC Grant 98-2113M-020-002-MY2. The authors gratefully acknowledge the MS technical assistance of Ms. Lan-Yu Wang of MST Co. Ltd.

\section{References}

[1] K. Ohtsubo and J. D. Marth, "Glycosylation in cellular mechanisms of health and disease," Cell, vol. 126, no. 5, pp. 855867, 2006.

[2] J. B. Lowe and J. D. Marth, "A genetic approach to mammalian glycan function," Annual Review of Biochemistry, vol. 72, pp. 643-691, 2003.

[3] M. Refaei, R. Leventis, and J. R. Silvius, "Assessment of the roles of ordered lipid microdomains in post-endocytic trafficking of glycosyl-phosphatidylinositol-anchored proteins in mammalian fibroblasts," Traffic, vol. 12, no. 8, pp. 1012-1024, 2011.

[4] M. Taverna, N. T. Tran, T. Merry, E. Horvath, and D. Ferner, "Electrophoretic methods for process monitoring and the quality assessment of recombinant glycoproteins," Electrophoresis, vol. 19, no. 15, pp. 2572-2594, 1998.

[5] A. Helenius and M. Aebi, "Intracellular functions of N-linked glycans," Science, vol. 291, no. 5512, pp. 2364-2369, 2001.

[6] J. B. Lowe, "Glycosylation, immunity, and autoimmunity," Cell, vol. 104, no. 6, pp. 809-812, 2001.

[7] T. H. Steinberg, B. J. Agnew, K. R. Gee et al., "Global quantitative phosphoprotein analysis using multiplexed proteomics technology," Proteomics, vol. 3, no. 7, pp. 1128-1144, 2003.

[8] N. Packer, M. Ball, and P. Devine, Methods in Molecular Biology, vol. 112, Humana Press, New Jersey, NJ, USA, 1999.
[9] M. Furlan, B. A. Perret, and E. A. Beck, "Staining of glycoproteins in polyacrylamide and agarose gels with fluorescent lectins," Analytical Biochemistry, vol. 96, no. 1, pp. 208-214, 1979.

[10] G. Muñoz, S. Marshall, M. Cabrera, and A. Horvat, "Enhanced detection of glycoproteins in polyacrylamide gels," Analytical Biochemistry, vol. 170, no. 2, pp. 491-494, 1988.

[11] E. A. Bayer, H. Ben-Hur, and M. Wilchek, "Enzyme-based detection of glycoproteins on blot transfers using avidinbiotin technology," Analytical Biochemistry, vol. 161, no. 1, pp. 123-131, 1987.

[12] I. Bouchez-Mahlout, C. Doyen, and M. Laurière, "Accurate detection of both glycoproteins and total proteins on blots: control of side reactions occurring after periodate oxidation of proteins," Electrophoresis, vol. 20, no. 7, pp. 1412-1417, 1999.

[13] T. H. Steinberg, K. Pretty, K. N. Berggren et al., "Rapid and simple single nanogram detection of glycoproteins in polyacrylamide gels and on electroblots," Proteomics, vol. 1, no. 7, pp. 841-855, 2001.

[14] R. M. Zacharius, T. E. Zell, J. H. Morrison, and J. J. Woodlock, "Glycoprotein staining following electrophoresis on acrylamide gels," Analytical Biochemistry, vol. 30, no. 1, pp. 148$152,1969$.

[15] A. H. Wardi and G. A. Michos, "Alcian blue staining of glycoproteins in acrylamide disc electrophoresis," Analytical Biochemistry, vol. 49, no. 2, pp. 607-609, 1972.

[16] A. E. Eckhardt, C. E. Hayes, and I. J. Goldstein, "A sensitive fluorescent method for the detection of glycoproteins in polyacrylamide gels," Analytical Biochemistry, vol. 73, no. 1, pp. 192-197, 1976.

[17] C. Hart, B. Schulenberg, T. H. Steinberg, W. Y. Leung, and W. F. Patton, "Detection of glycoproteins in polyacrylamide gels and on electroblots using Pro-Q Emerald 488 dye, a fluorescent periodate Schiff-base stain," Electrophoresis, vol. 24, no. 4, pp. 588-598, 2003.

[18] L. B. Silverman-Gavrila, A. G. Senzel, M. P. Charlton, and Z.-P. Feng, "Expression, phosphorylation, and glycosylation of CNS proteins in aversive operant conditioning associated memory in Lymnaea stagnalis," Neuroscience, vol. 186, pp. 94109, 2011.

[19] J. You, A. Fitzgerald, P. J. Cozzi et al., "Post-translation modification of proteins in tears," Electrophoresis, vol. 31, no. 11, pp. 1853-1861, 2010.

[20] G. T. Hermanson, Bioconjugate Techniques, Academic Press, San Diego, Calif, USA, 2nd edition, 2008.

[21] Z. Zhao, Y. Aliwarga, and M. D. Willcox, "Intrinsic protein fluorescence interferes with detection of tear glycoproteins in SDS-polyacrylamide gels using extrinsic fluorescent dyes," Journal of Biomolecular Techniques, vol. 18, no. 5, pp. 331-335, 2007.

[22] R. L. Easton, M. S. Patankar, G. F. Clark, H. R. Morris, and A. Dell, "Pregnancy-associated changes in the glycosylation of tamm-horsfall glycoprotein: expression of sialyl lewis sequences on core 2 type o-glycans derived from uromodulin," The Journal of Biological Chemistry, vol. 275, no. 29, pp. 21928-21938, 2000. 

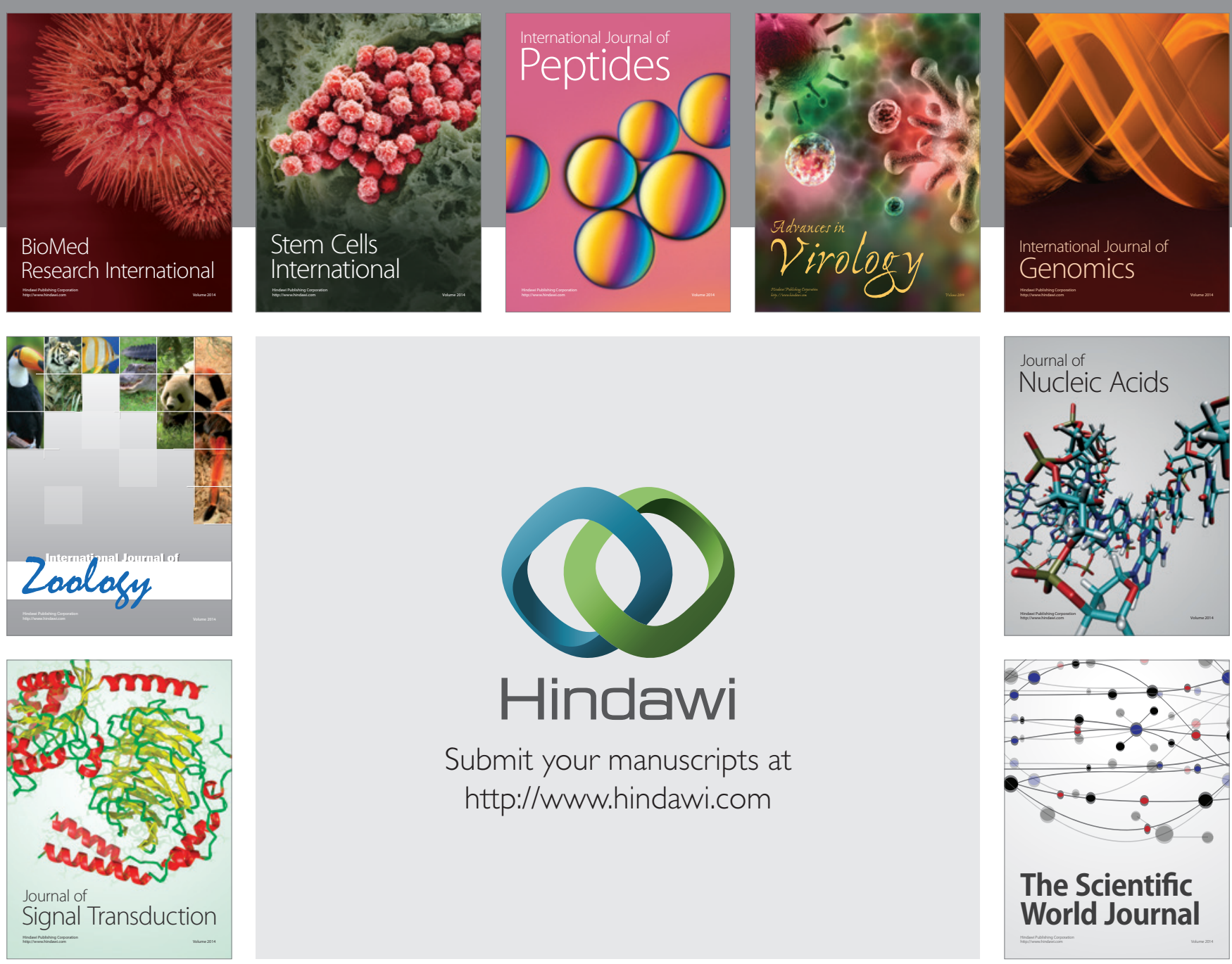

Submit your manuscripts at

http://www.hindawi.com
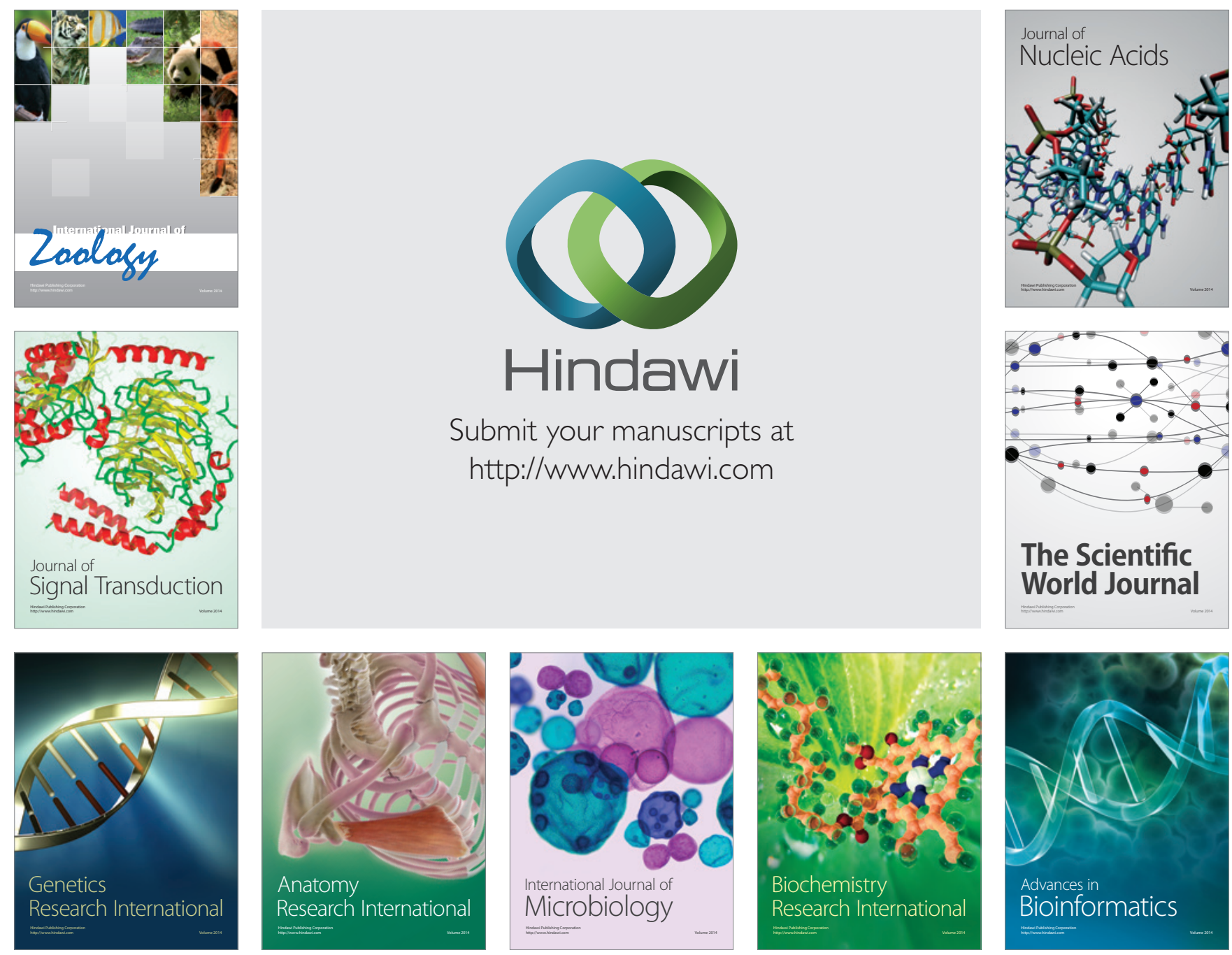

The Scientific World Journal
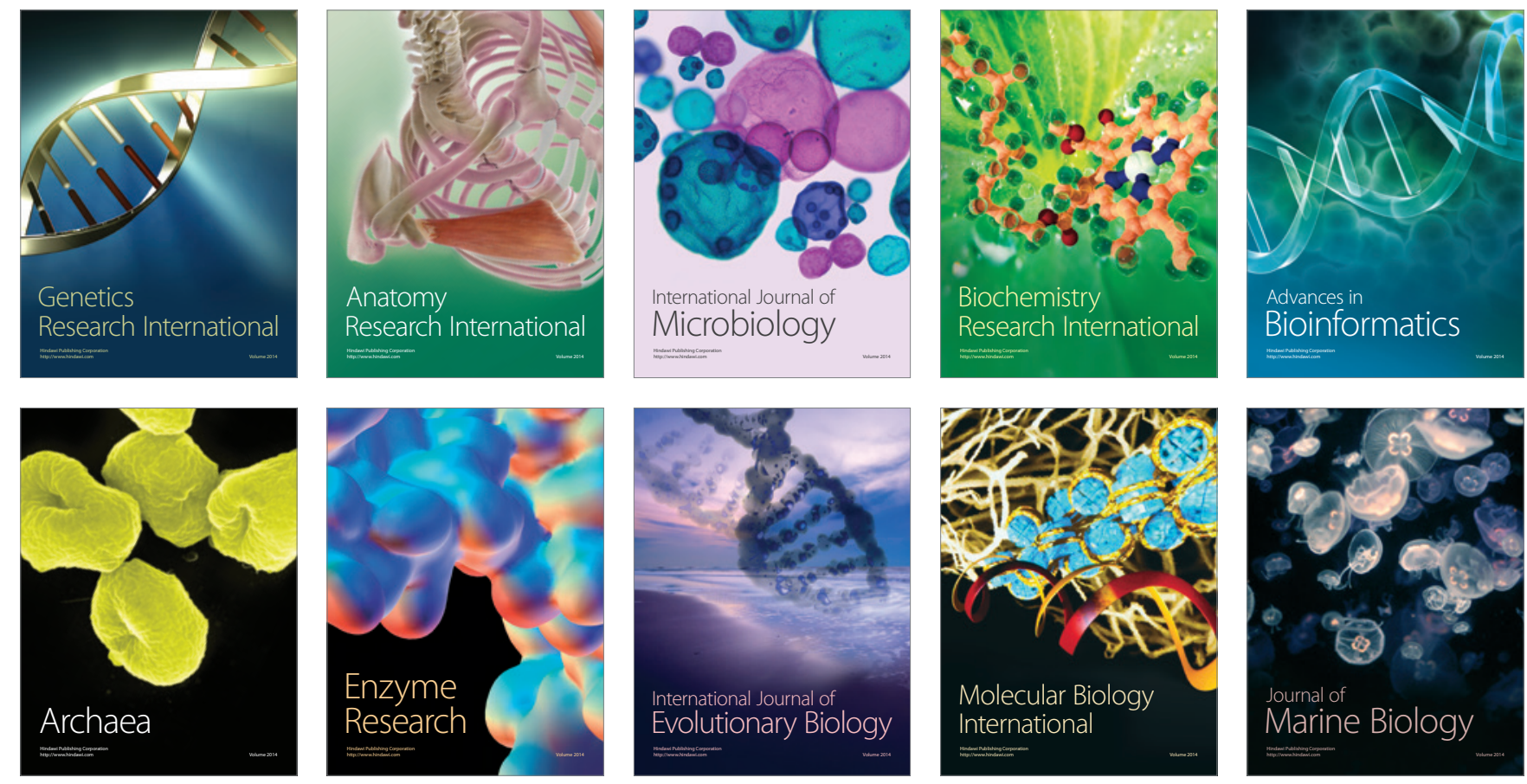